\title{
Simulation of the head-tail instability of colliding bunches
}

\author{
E. A. Perevedentsev and A. A. Valishev \\ Budker Institute for Nuclear Physics, Novosibirsk, 630090, Russia \\ (Received 22 October 2000; published 15 February 2001)
}

\begin{abstract}
A numerical simulation of a possible coherent beam-beam instability of the head-tail type is presented. The studied model employs a linearized coherent beam-beam interaction and takes complete account of the bunch length, including the deformation of the colliding bunches during their overlap. Measured head-tail mode spectra from the VEPP-2M collider are compared with the results of the analytical model and tracking. A computer code for evaluation of the coherent mode increments, which was used for analysis of possible ways to stabilize the beam-beam system, is described.
\end{abstract}

DOI: 10.1103/PhysRevSTAB.4.024403

\section{INTRODUCTION}

Beam-beam effects, which are the main phenomena limiting the luminosity of modern colliders, have been the focus of accelerator physicists' attention for a long time [1]. Analytic equations describing processes happening in the colliding beam systems are extremely complex because of its intrinsic nonlinearity. Therefore a computer simulation using particle tracking becomes an important tool for analysis of the beam-beam system. Another way to solve the problem is by system simplification; in particular, for small betatron oscillation amplitudes, it is often allowable to linearize the transverse force exerted by the beams on each other. This case is referred to as the linearized beam-beam interaction. Both the rigid transverse distribution model [1,2] and the solution of the linearized Vlasov equation [3-5] for two beams of equal intensities predict the existence of two coherent beam-beam modes: the $\sigma$ mode with the tune equal to the betatron tune $\nu_{\beta}$, and the $\pi$ mode with the tune shifted by $Y \xi$, where $\xi$ is the beam-beam parameter and $Y$ is a factor approximately equal to $1[2,5]$. These modes have been observed in different machines $[1,6]$.

The single bunch collective phenomena including the head-tail effect are well known [7]. The latter arises from the interplay of longitudinal and transverse motion and is expressed in terms of coherent synchrobetatron modes coupled via transverse wakefields. Usually the betatron coupling between 2 transverse degrees of freedom is small, and therefore a separate treatment of horizontal and vertical synchrobetatron oscillations is a good approximation. Since the rise time of the head-tail instability is much shorter than the radiation damping time in $e^{+} e^{-}$machines, we can drop the radiative effects in what follows.

The residual coherent beam-beam interaction from separated $e^{+} e^{-}$beams is known to enhance the head-tail instability caused by the transverse impedance in the LEP collider [8], and numerical studies of this combined effect were done using a model where the bunch length was neglected in calculating the beam-beam kick, i.e., for the case of a bunch much shorter than the beta-function value, $\beta^{*}$, at the interaction point (IP) [9].

$1098-4402 / 01 / 4(2) / 024403(8) \$ 15.00$
PACS numbers: 29.27.Bd, 29.20.-c

It has been recently proposed that a finite length (i.e., $\sim \beta^{*}$ ) of colliding bunches can lead to the coupling of synchrobetatron modes in the beam-beam system, and thus a head-tail-type instability of colliding bunches may arise [10]. In the case of two circulating colliding bunches, which are assumed to produce no wakefields other than those due to the collective beam-beam response, this linear model does not predict any instability unless one of the mode tunes reaches a resonance. However, a head-tail instability appears in the beam-beam system if the transverse impedance of the circular collider is taken into consideration, or in the special case of a circulating bunch colliding with a bunch produced anew on each turn (a so-called linac-ring collision).

In this paper we study the frequency spectrum of the synchrobetatron oscillations of colliding bunches. The linearized beam-beam force model is used in our calculations, which include the bunch length effect resulting in the fact that the colliding bunches are bent during their overlap. Owing to the linearized beam-beam approximation, the dynamics of the two transverse dimensions decouple, hence it is permissible to consider only one such dimension. The coherent mode spectrum is obtained using two methods: an analytical method based on the circulant matrix formalism and numerical particle tracking. Section II describes the circulant matrix method, while the model used in the particle tracking is presented in Sec. III. In Sec. IV we discuss the results for the beam-beam interaction in a collider free of impedance elements and compare the calculations with experimental data. In Sec. V the effect of machine impedance is considered. Section VI gives the results for a linac-ring collision scheme.

\section{CIRCULANT MATRIX METHOD}

The detailed theory of the circulant matrix describing the collective synchrobetatron motion is given in Ref. [11]. Here we shall focus only on an extension of the method to the case of two colliding bunches, aiming at a spectral analysis of the dipole synchrobetatron modes of the beambeam system. 
We use the so-called "hollow beam" model. It assumes that all particles of the bunch have equal synchrotron amplitudes and are evenly spread over the synchrotron phase, forming a ring in the synchrotron phase space. The ring is divided into $N$ mesh elements, each characterized by its transverse dipole moment and its synchrotron phase. The dipole moment of the $i$ th mesh, $1<i<N$, is proportional to the transverse displacement $x_{i}$ of the centroid of the particles populating this mesh, times the portion $N_{b} / N$ of the bunch intensity, $N_{b}$, per mesh. The betatron motion will be described in terms of the normalized betatron variables, $x_{i}$ and $p_{i}$, where $p_{i}$ is the respective momentum. Thus $2 N$ variables will be needed to characterize synchrobetatron motion in each bunch. They form a $2 N$ vector, where $x_{i}$ and $p_{i}$ are listed in the order corresponding to the mesh number, according to its synchrotron phase.

The synchrobetatron oscillations of $N$ elements forming a bunch are represented by the $2 N \times 2 N$ matrix $M$, which maps the above vector over the collider arc,

$$
M=C \otimes B,
$$

where $\otimes$ denotes the outer product, $B$ is the usual betatron oscillation matrix

$$
B=\left(\begin{array}{cc}
\cos \mu_{\beta} & \sin \mu_{\beta} \\
-\sin \mu_{\beta} & \cos \mu_{\beta}
\end{array}\right),
$$

$C$ is the $N \times N$ circulant matrix [11] with elements

$$
\begin{aligned}
C_{i j}= & \frac{\sin N \varphi_{i j}}{N \sin \varphi_{i j}}, \\
\varphi_{i j}= & \frac{1}{2}\left(\mu_{s}-(N-i+j) \frac{2 \pi}{N}\right), \\
& (1<i, j<N),
\end{aligned}
$$

and $\mu_{\beta}, \mu_{s}$ are the betatron and synchrotron phase advances. With $N=2 m+1$, the eigenvectors and eigenvalues of matrix $M$ exactly correspond to the first $-m, \ldots, m$ synchrobetatron harmonics with the tunes $\nu_{\beta}-m \nu_{s}, \ldots, \nu_{\beta}+m \nu_{s}, \nu_{\beta, s}=\mu_{\beta, s} / 2 \pi$.

Note that the synchrotron oscillation in the circulant matrix formalism transports the dipole moment values around the circle formed by the mesh elements with fixed synchrotron phases (i.e., fixed longitudinal positions in the bunch), rather than performing a permutation of the meshes themselves.

Expansion of the model to the case of two noninteracting bunches is straightforward by using a $4 N \times 4 N$ matrix,

$$
M_{2}=\left(\begin{array}{ll}
1 & 0 \\
0 & 1
\end{array}\right) \otimes M
$$

The linearized beam-beam interaction is described by a $4 N \times 4 N$ matrix $M_{b b}$ consisting of consecutive short

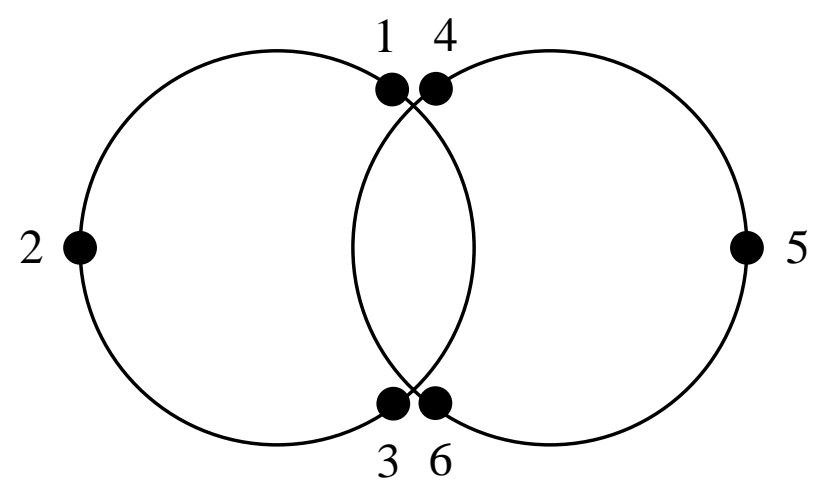

FIG. 1. Position of macroparticles in the synchrotron phase space.

kicks and drifts between interactions of macroparticles sitting in each mesh, and assumed to be rigid Gaussian disks [2]. For example, let us consider the interaction of two bunches each consisting of three elements. Since matrix $M_{2}$ makes a transformation from the IP to the IP, the first step is the longitudinal unfolding of the bunch. Figure 1 shows the position of the particles in their mesh elements before the first interaction. The next step is the interaction between particles $1,3,4$, and 6 , which is linear in relative distance. For instance, the kick given to the first particle is

$$
\Delta p_{1}=-\frac{2 \pi \xi}{3}\left[\left(x_{1}-x_{6}\right)+\left(x_{1}-x_{4}\right)\right]
$$

where $x$ and $p$ are the particle's coordinate and momentum, and $\xi$ is the beam-beam parameter. Next follows the free drift and interaction of particles 2, 4, 6 and 1,3,5; next the drift and interaction of the "tail" particles No. 2 and No. 5, and finally longitudinal folding of the bunch to the IP. Generalization of the algorithm to the case of $N>3$ is evident and can be left for the reader.

The complete one-turn matrix $M_{t}$ is the product of the arc matrix and the beam-beam matrix, $M_{t}=M_{2} M_{b b}$. Its eigenvalues and eigenvectors completely characterize the synchrobetatron modes of the beam-beam system and can be obtained numerically using a computer algebra system.

The above analytical approach is not intended for obtaining closed-form solutions, rather it provides an algorithm, which proved efficient for $N<10$.

\section{PARTICLE TRACKING}

\section{A. Tracking algorithm}

As compared with the analytical method, straightforward tracking is easily implemented for a large number $N_{p}$ of macroparticles and is not limited to the hollowbeam model; we can use any particle distribution in the synchrotron phase space of a bunch. However, comparisons of simulations for the hollow and filled beam have shown no significant difference in the synchrobetatron 
mode spectrum. ${ }^{1}$ A large number of macroparticles participating in the simulation is required to evaluate the amplitudes and frequencies of higher-order modes, but usually these modes are not of much importance because of small coupling. Moreover, they are suppressed by quantum fluctuations of the synchrotron radiation. These factors, together with an increase of the tracking time, set the reasonable limit of the $N_{p}$ value at about 50 .

The tracking scheme is presented in Fig. 2. First, the initial conditions for all macroparticles of the two bunches are set: $x_{1, i}, x_{2, j}, p_{1, i}, p_{2, j}, s_{1, i}, s_{2, j}, \delta_{1, i}$, and $\delta_{2, j}$. Here subscripts $1, i$ and $2, j\left(i, j=0, \ldots, N_{p}\right)$ label betatron coordinates $x$, momenta $p$, longitudinal coordinates $s$, and energy deviations $\delta$ of macroparticles in the two bunches. Typically, the initial displacements and momenta in one bunch were set to zero while the other had some small initial offset. The offset value is not significant because all interactions are linear in $x$. Stage II is sorting the particles in each bunch so that the larger numbers $i, j$ would label the larger $s$, corresponding to the bunch tail. This allows us to establish the correct interaction sequence and to calculate the longitudinal coordinate of the interaction point $\Delta s_{i j}$ of particles $i, j$ with respect to the reference at the IP. Next, in stage III we unfold the bunches, i.e., we calculate the particles' betatron displacements at $\Delta s_{i j}$ using their initial momenta, as if there were no fields in the interaction region:

$$
\begin{aligned}
& \tilde{x}_{1, i}=x_{1, i}-p_{1, i} \Delta s_{i j}, \\
& \tilde{x}_{2, j}=x_{2, j}+p_{2, j} \Delta s_{i j} .
\end{aligned}
$$

Stage IV gives the change in momenta due to the beambeam kick,

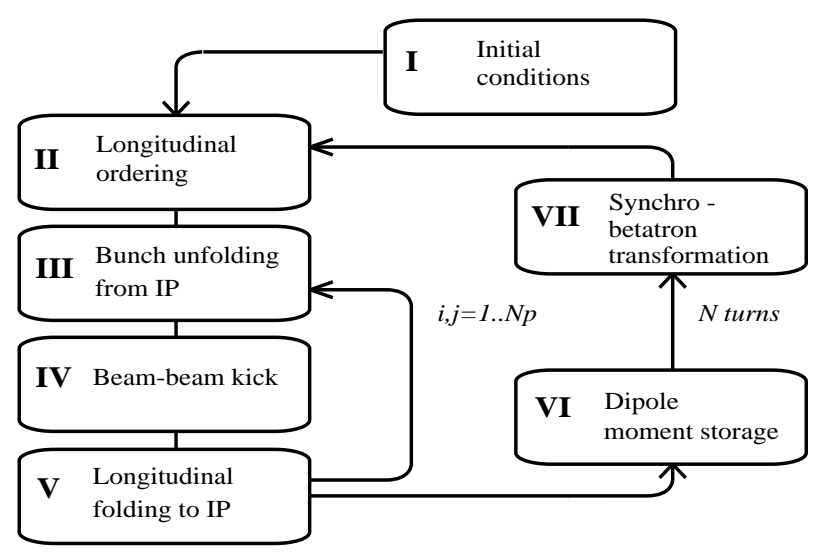

FIG. 2. Tracking process chart.

\footnotetext{
${ }^{1}$ Typically, the radial modes in the head-tail mode spectrum are not important for the bunch length shorter than the wake variation length, which is our case.
}

$$
\begin{aligned}
\Delta p_{1, i} & =-\frac{2 \pi \xi}{N_{p}}\left(\tilde{x}_{1, i}-\tilde{x}_{2, j}\right), \\
\tilde{p}_{1, i} & =p_{1, i}+\Delta p_{1, i}, \\
\Delta p_{2, j} & =-\frac{2 \pi \xi}{N_{p}}\left(\tilde{x}_{2, j}-\tilde{x}_{1, i}\right), \\
\tilde{p}_{2, j} & =p_{2, j}+\Delta p_{2, j} .
\end{aligned}
$$

Here we assume that the bunches have equal intensities and the kick does not change over the bunch length, which implies that the bunch length is less than $\beta^{*}$. Stage V returns the particles to the reference point at the IP,

$$
\begin{aligned}
& x_{1, i}=\tilde{x}_{1, i}+\tilde{p}_{1, i} \Delta s_{i j}, \\
& x_{2, j}=\tilde{x}_{2, j}-\tilde{p}_{2, j} \Delta s_{i j} .
\end{aligned}
$$

Summation of the resulting displacements over $i$ and $j$ gives the total dipole moments of each bunch to be stored in stage VI. The one-turn iteration loop is closed with stage VII, which performs the linear synchrobetatron transformation

$$
\begin{aligned}
& \left(\begin{array}{l}
x \\
p
\end{array}\right)_{1, i}^{\prime}=\left(\begin{array}{cc}
\cos \mu_{\beta} & \sin \mu_{\beta} \\
-\sin \mu_{\beta} & \cos \mu_{\beta}
\end{array}\right)\left(\begin{array}{l}
x \\
p
\end{array}\right)_{1, i}, \\
& \left(\begin{array}{l}
s \\
\delta
\end{array}\right)_{1, i}^{\prime}=\left(\begin{array}{cc}
\cos \mu_{s} & \sin \mu_{s} \\
-\sin \mu_{s} & \cos \mu_{s}
\end{array}\right)\left(\begin{array}{l}
s \\
\delta
\end{array}\right)_{1, i} .
\end{aligned}
$$

Here we use the normalized betatron coordinates $x=$ $X / \sqrt{\beta^{*}}$ and $p=P \sqrt{\beta^{*}}$.

The betatron tune chromaticity, $\chi$, can be taken into consideration in this stage by including it in the betatron phase advance:

$$
\mu_{\beta}=2 \pi\left(\nu_{\beta}+\chi \delta\right) .
$$

\section{B. Data postprocessing}

The spectrum of coherent modes was obtained by applying the Fourier transform to the array of dipole moments sampled on each turn. In the Fourier amplitudes, peaks higher than a predefined value were defined as reliable and their tune was evaluated. The interpolated fast-Fourier transform with the Hanning data windowing [12] was used to improve the tune determination accuracy.

In general, some synchrobetatron modes may show exponential growth or decay, with their characteristic times and respective increments or decrements, bringing time-dependent amplitudes in the total dipole moment. Our increment calculation method is based on the time dependence of the Fourier peaks. Consider a sinusoidal signal with an exponentially changing amplitude

$$
S(t)=A e^{\lambda\left(t_{0}+t\right)} \cos \omega t .
$$

For small $\lambda$, the Fourier amplitude of harmonic $\omega$ is

$$
A_{F} \approx A e^{\lambda\left(t_{0}+T_{w} / 2\right)} \frac{\sinh \lambda T_{w} / 2}{\lambda T_{w}},
$$


where $T_{w}$ is the time-window width. A Fourier transform of two data windows with the same width, one with $t_{0}=0$, the other with $t_{0}=T$, gives the amplitudes $A_{F}(0)$ and $A_{F}(T)$. From these, the value of increment, $\lambda$, can be obtained:

$$
\lambda=\frac{1}{T} \ln \frac{A_{F}(T)}{A_{F}(0)} .
$$

This technique can also be applied in the case of multiple harmonics. Evaluation of $\lambda$ becomes difficult when some of the modes are unstable, so that excessive growth of their Fourier amplitudes can suppress contributions of stable and damped modes. This can cause an error due to noise contribution to the small amplitudes. Therefore, the spectrum calculation in more than two windows is needed to improve accuracy of the evaluation of the mode increments using the least squares method.

\section{SYNCHROBETATRON MODES OF THE BEAM-BEAM SYSTEM}

Figure 3 presents calculations of the mode spectrum vs the beam-beam parameter for the head-on collision of equal intensity bunches circulating with the same betatron and synchrotron tunes. Dashed lines show the matrix method results with $N=5$; circles are the tracking data for 50 particles per bunch. Three horizontal lines show the $\nu_{\beta}-\nu_{s}, \nu_{\beta}$, and $\nu_{\beta}+\nu_{s}$ tune values. A good agreement is seen up to $\xi=2 \nu_{s}$, with only small differences at higher bunch intensities. These differences are due to a limited number of synchrobetatron modes considered in the matrix model $(m=-2,-1,0,+1,+2)$, which is insufficient to describe coupling with higher-order modes.
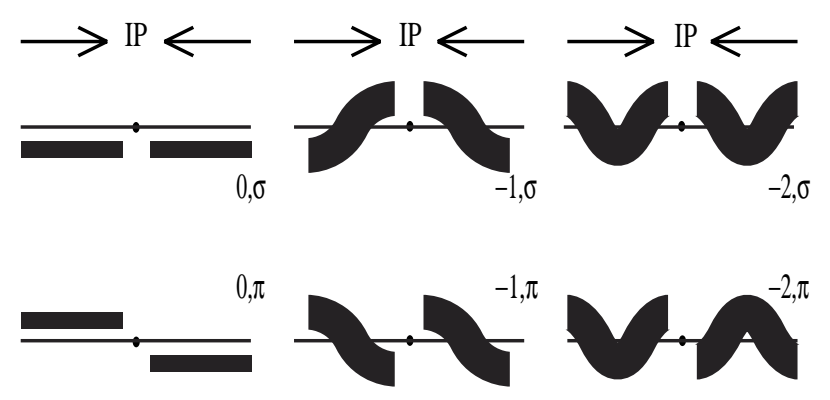

FIG. 4. Schematic view of the coherent synchrobetatron beambeam modes.

For identification of harmonics in Fig. 3 we introduced a notation illustrated in Fig. 4. The first index in the notation labels the synchrotron wave number, i.e., the number of dipole moment variations over the synchrotron phase. The second index, $\sigma$ or $\pi$, is borrowed from the rigid beam model and labels the coherent beam-beam eigenmodes with even and odd symmetries between the two colliding bunches, respectively. We can see that degeneracy of $m \sigma$ and $m \pi$ modes $(m \neq 0)$ is removed by the mode coupling when the mode tunes approach each other.

The same calculation for colliding bunches of unequal intensity $\left(\xi_{1} / \xi_{2}=0.5\right)$ is presented in Fig. 5, where more modes seem to be exhibited simply because the intensity asymmetry completely removes degeneracy from the mode spectrum.

In perfect agreement with the theoretical model [10], these calculations have shown that the mode tunes do not intersect. Their repulsion is due to coupling of the synchrobetatron modes via the collective beam-beam response. There is no coherent instability in the whole range of $\xi$

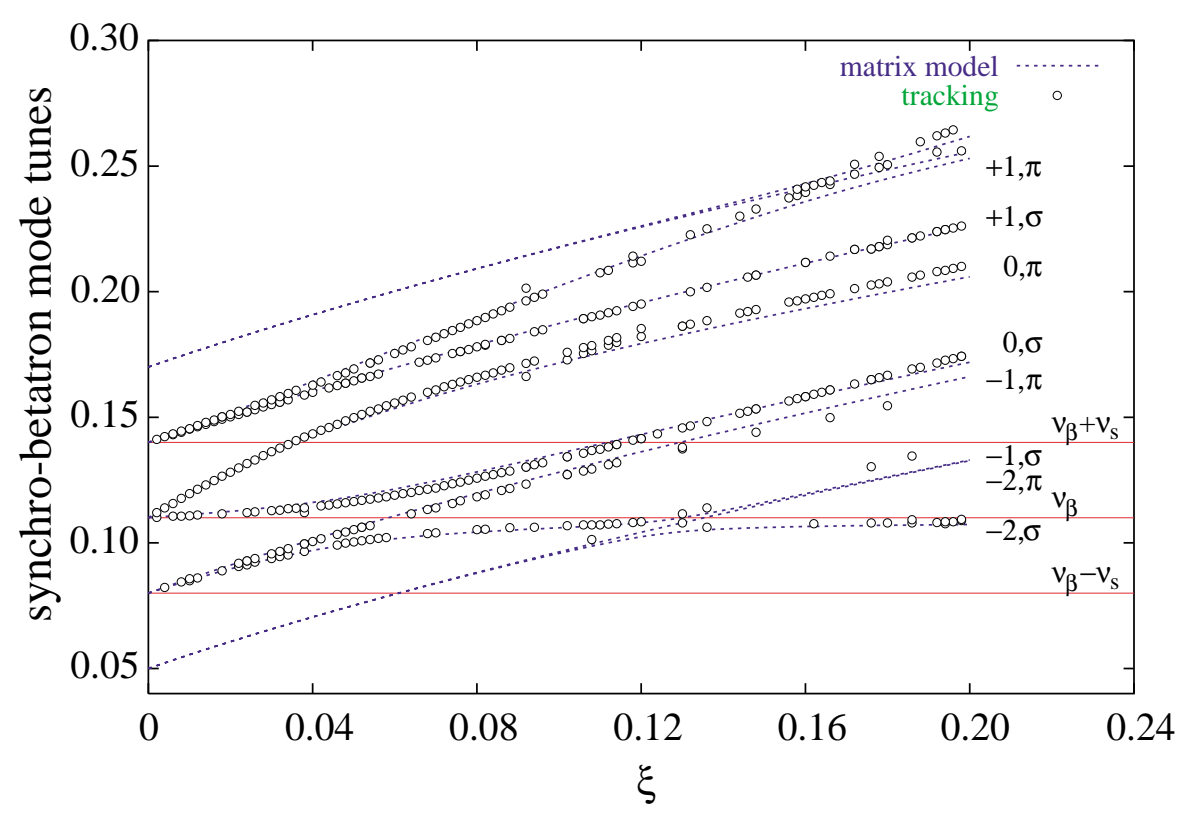

FIG. 3. (Color) Synchrobetatron mode tunes vs $\xi$. Comparison of the circulant matrix model and tracking. Equal bunch intensities, $\nu_{\beta}=0.11, \nu_{s}=0.03$, and the bunch length is $0.7 \beta^{*}$. 


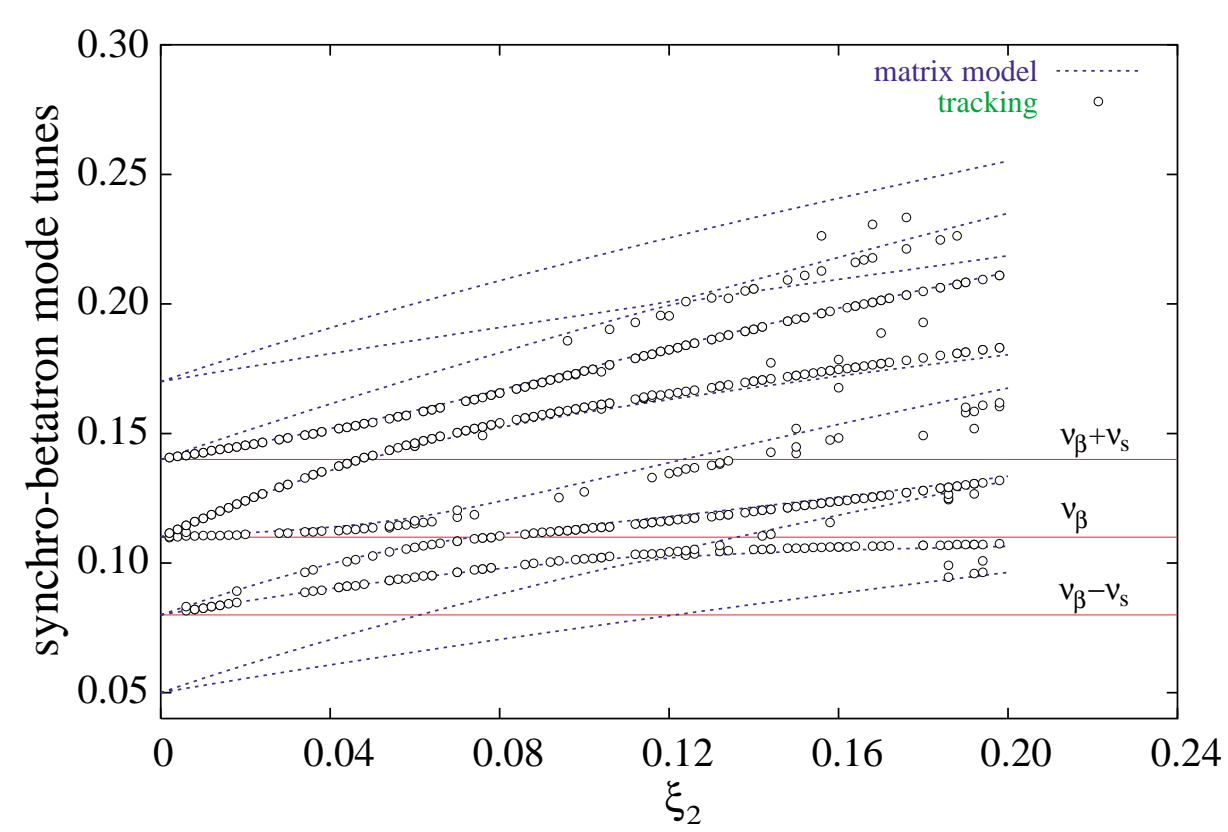

FIG. 5. (Color) Synchrobetatron mode tunes vs $\xi_{2}$. Comparison of the circulant matrix model and tracking. Unequal bunch intensities, $\xi_{1} / \xi_{2}=0.5, \nu_{\beta}=0.11, \nu_{s}=0.03$, and the bunch length is $0.7 \beta^{*}$.

when neither mode reaches the tune of 0 or 0.5 . Interaction in this system is symmetrical, and the system is closed. Oscillations in such systems are stable.

Since the VEPP-2M collider (BINP, Novosibirsk) [13] has a negligible transverse impedance, we had an opportunity to compare these simulation results with experimental data. Vertical oscillations of the electron bunch were excited with a short kicker pulse, and following coherent oscillations of the two bunches were observed. Vertical coordinates of the bunches were sampled by a fast analogto-digital converter at 8192 turns. The Fourier transform of the collected data gave the coherent modes spectrum, where the proposed synchrobetatron modes of the beam-beam system were experimentally detected, and their

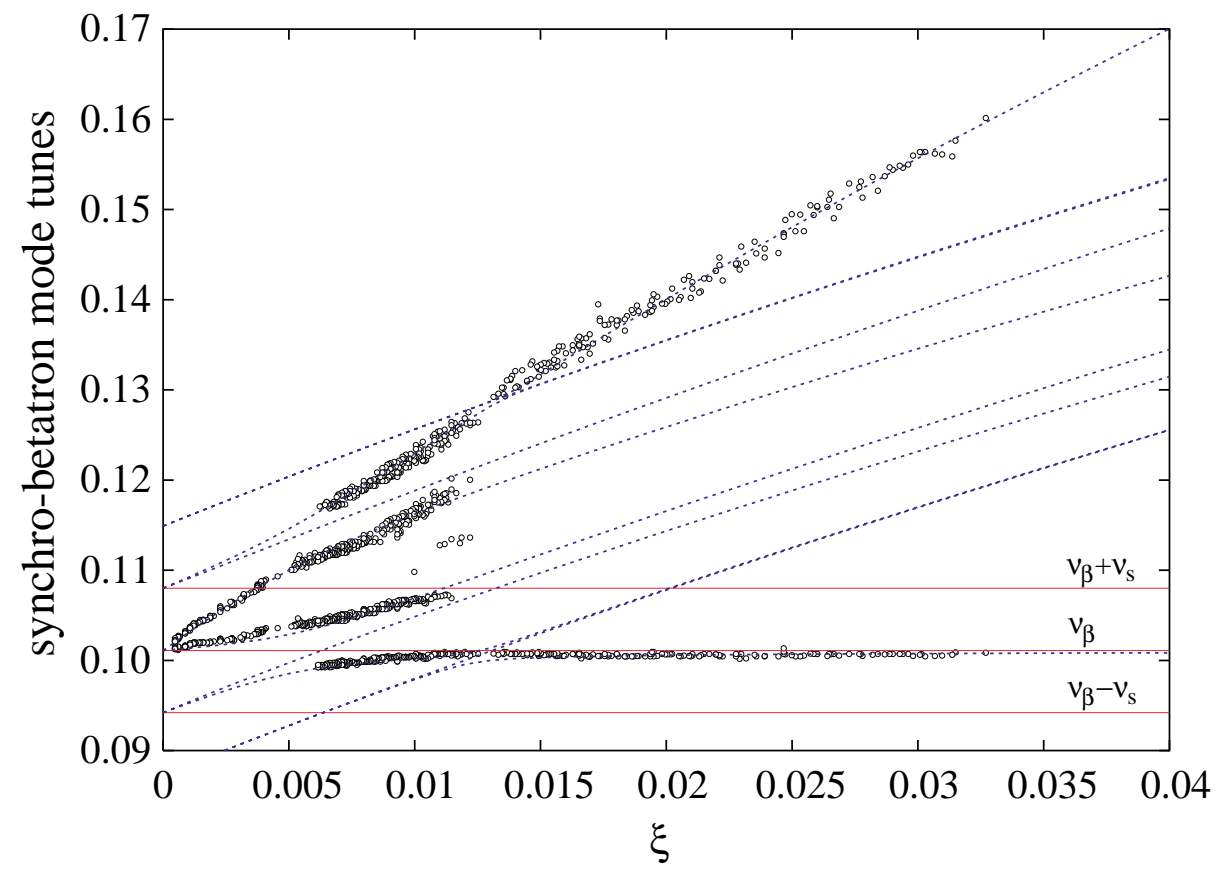

FIG. 6. (Color) Synchrobetatron mode tunes vs the beam-beam parameter $\xi$ per one IP. Experimental data (circles) and simulation (lines). VEPP-2M was operated with one $e^{+}$and one $e^{-}$bunch colliding at two IPs, $\nu_{\beta}=0.101, \nu_{s}=0.0069, \beta^{*}=6 \mathrm{~cm}$, and the bunch length was $3.5 \mathrm{~cm}$. 
spectrum was measured as a function of the beam-beam parameter at different synchrotron tunes. Figure 6 shows that our model provides an excellent agreement with the experiment [14]. This agreement corroborates the analytical and numerical models employed, and validates their extension to the case where the transverse impedance in the machine is not small.

\section{IMPEDANCE}

The action of the machine impedance is introduced in the tracking code by adding the collective kick to each bunch. The kick of the $i$ th particle is then

$$
\Delta p_{i}=\sum_{j=1}^{i-1} Q x_{j},
$$

where, for simplicity, a constant wake model is used, which is adequate for a short bunch. Generalization for any time dependence of the transverse wakefield is straightforward.

This collective interaction makes the system nonHamiltonian and its behavior changes drastically: the coherent instability of colliding bunches appears without a threshold. Exponential growth of the mode amplitude is characterized by the mode increment per turn, defined in Sec. IIIB. Figure 7 shows the dependence of the mode increments on $\xi$ for the constant wake. A negative increment means damping. The increments are linear in impedance.

The betatron tune chromaticity, $\chi$, causing an additional adjustable coupling of transverse and longitudinal motion, is a common cure for this type of instability. Consideration of the chromaticity effect has shown that the low-order synchrobetatron modes, $m=0,-1$, either $\sigma$ or $\pi$, can be damped, whereas other modes remain unstable and their dependence on $\chi$ is very complicated [10].

\section{LINAC-RING COLLISIONS}

A special scheme of the colliding beams has been recently proposed for experiments on electron interaction with heavy ions: to collide the ion bunches circulating in a storage ring with the electron bunches supplied by a linac. This is called the linac-ring collision scheme.

In each collision particles of the electron bunch have zero coordinates and momenta and the ion bunch preserves memory about previous interactions. In the tracking algorithm this is simply achieved by setting all $x_{i}$ and $p_{i}$ of one bunch to zero on each turn. For the linac-ring scheme, the matrix model can be modified by using matrix $M_{l c}$,

$$
M_{l c}=\left(\begin{array}{cc}
1 & 0 \\
0 & 0
\end{array}\right) \otimes M
$$

instead of $M_{2}$.

In the simulation we take equal beam-beam parameters, $\xi$, in the electron and ion beams, and both colliding bunches' lengths are set equal to $\beta^{*}$; in the ion ring $\nu_{\beta}=0.1, \nu_{s}=0.002$, and the momentum deviation amplitude is assumed to be equal to $\nu_{s}$, so that the ion ring chromaticity, $\chi$, numerically coincides with the chromatic phase.

In the linac-ring collision scheme the head-tail instability results from the betatron phase advance over the

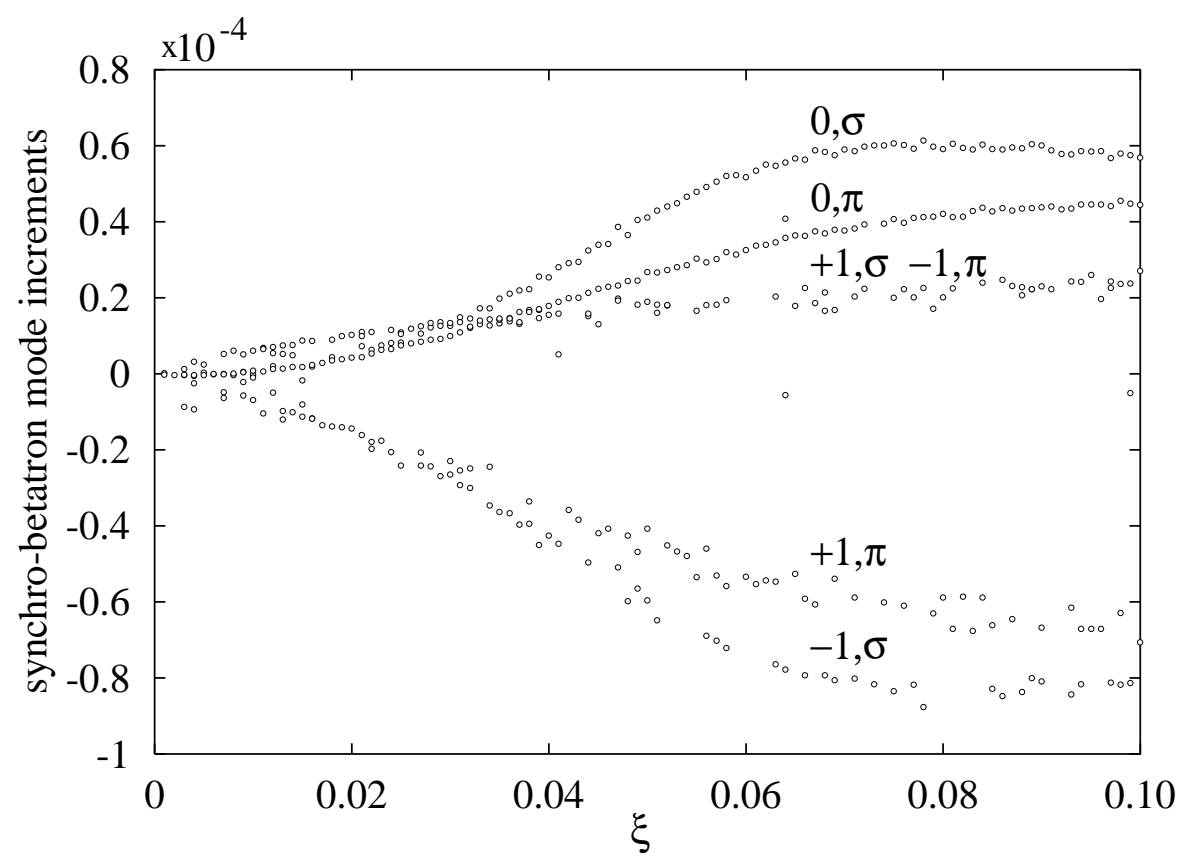

FIG. 7. Synchrobetatron mode increments per turn vs $\xi$ for combined action of the beam-beam interaction and machine impedance (tracking). Equal bunch intensities, $\nu_{\beta}=0.11, \nu_{s}=0.03$, and the bunch length is $0.7 \beta^{*}$. The constant wake, $Q=0.005$, corresponds to the $m=0$ mode tuneshift of $-3 \times 10^{-5}=-10^{-3} \nu_{s}$. 


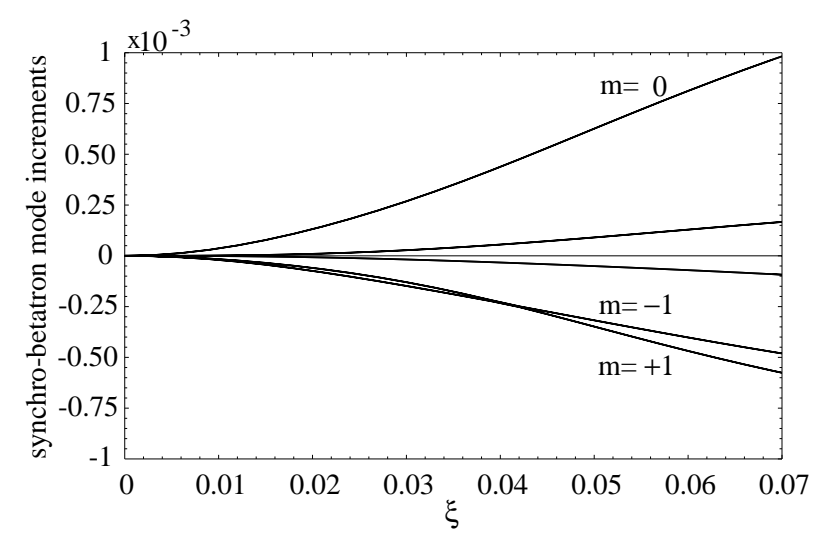

FIG. 8. Synchrobetatron mode increments per turn vs $\xi$. The linac-ring scheme, equal beam-beam parameters in both the beams, $\nu_{\beta}=0.1, \nu_{s}=0.002$, and the bunch length is equal to $\beta^{*}$.

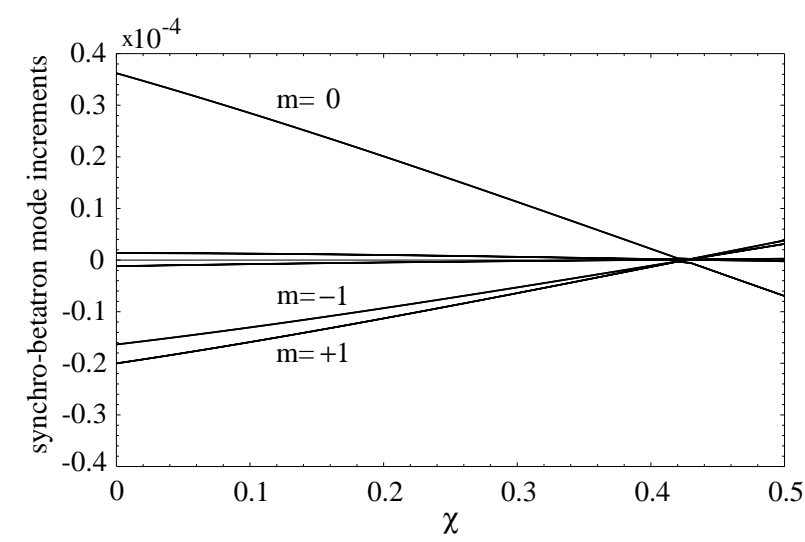

FIG. 9. Synchrobetatron mode increments per turn vs the chromaticity $\chi$ with constant $\xi=0.01$; other parameters are the same as in Fig. 8.

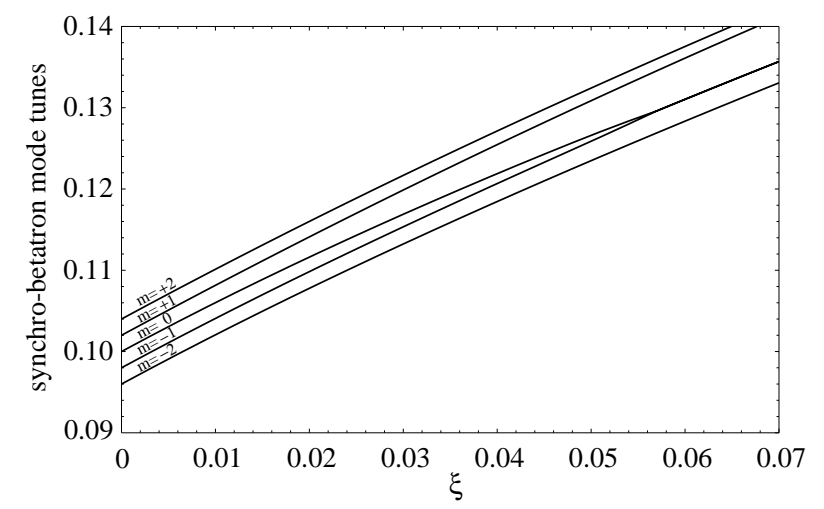

FIG. 10. Synchrobetatron mode tunes vs $\xi$ for constant chromaticity $\chi=0.409$; other parameters are the same as in Fig. 8.

beam-beam interaction length, but this effect can be exactly compensated by the ion ring chromaticity. For zero chromaticity some modes are unstable (Fig. 8), but it is possible to stabilize all of them at a certain positive value of $\chi$ (Fig. 9). This value does not depend on the beam-beam parameter $\xi$, so this is a perfect cure for the instability.

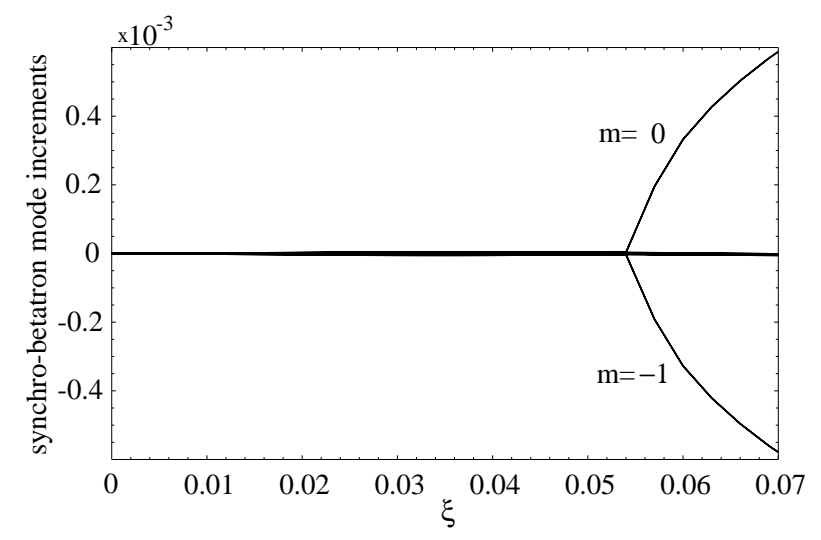

FIG. 11. Synchrobetatron mode increments per turn vs $\xi$ for constant chromaticity $\chi=0.409$; other parameters are the same as in Fig. 8.

When the phase advance over the bunch length is compensated by the chromaticity, the 0 and -1 modes couple as in the strong head-tail effect. The instability in this case has a threshold up to which a normal operation is possible (Figs. 10 and 11).

\section{CONCLUSIONS}

Using the circulant matrix formalism and numerical simulation we studied the coherent synchrobetatron mode spectrum of colliding bunches of finite length for linearized beam-beam interaction. In both the approaches, the bunches are sliced into transversely rigid macroparticles. However, the bunches are not longitudinally rigid, and we take into account their deformation during the beam-beam overlap.

For the case of pure beam-beam interaction of circulating bunches, coherent oscillations always remain stable unless some of the mode tunes reach a resonance. The coherent synchrobetatron mode spectra predicted by both models for this case of negligible transverse impedance have been observed experimentally at the VEPP-2M collider. The measured data are in good quantitative agreement with our analytical and numerical models.

Calculations involving the machine impedance predict a coherent beam-beam instability without a threshold. Some, though not all, of the synchrobetatron modes can be damped by optimizing the betatron tune chromaticity. Since the models used the linearized beam-beam interaction, their prediction of instability is not as conclusive as the above prediction of stability.

The linac-ring collision examination has shown that a head-tail instability of the chromatic type is possible in such a beam-beam system without any impedance elements in the machine. By adjusting the ring chromaticity, complete stabilization of the system is possible up to the threshold of the strong head-tail instability, also caused by the beam-beam interaction. 


\section{ACKNOWLEDGMENTS}

We thank I. N. Nesterenko and A. A. Polunin for their help in the experimental part of the work. We are indebted to R. Noer for careful reading and correcting the manuscript.

[1] A. Piwinski, IEEE Trans. Nucl. Sci. 26, 4268 (1979).

[2] K. Hirata, Nucl. Instrum. Methods Phys. Res., Sect. A 269, 7 (1988).

[3] R. E. Meller and R. H. Siemann, IEEE Trans. Nucl. Sci. 28, 2431 (1981).

[4] A. W. Chao and R. D. Ruth, Part. Accel. 16, 201 (1985).

[5] K. Yokoya and H. Koiso, Part. Accel. 27, 181 (1990).

[6] H. Koiso, Y. Funakoshi, T. Kawamoto, E. Kikutani, H. Nakayama, H. Ozaki, R. Sugahara, and J. Urakawa, Part. Accel. 27, 83 (1990).

[7] A.W. Chao, Physics of Collective Beam Instabilities in High Energy Accelerators (Wiley, New York, 1993).

[8] K. Cornelis and M. Lamont, in Proceedings of the 4th European Particle Accelerators Conference, London, 1994 (World Scientific, Singapore, 1994), Vol. 2, p. 1150.
[9] K. Cornelis, CERN Report No. SL/Note 93-39(OP), 1993; in Proceedings of the Chamonix Workshop, 1994 (CERN Report No. SL/94-06, 1994), p. 185; G. X. Li and K. Cornelis, CERN Report No. SL/94-85 (AP), 1994.

[10] E. A. Perevedentsev, in Proceedings of the Particle Accelerator Conference, New York, 1999 (IEEE, Piscataway, NJ, 1999), Vol. 3, p. 1521; in Proceedings of the International Workshop on Performance Improvement of ElectronPositron Collider Particle Factories, Tsukuba, 1999 (KEK, Tsukuba, Japan, 2000), p. 171.

[11] V. V. Danilov and E. A. Perevedentsev, CERN Report No. SL/93-38 (AP), 1993; Nucl. Instrum. Methods Phys. Res., Sect. A 391, 77 (1997).

[12] R. Bartolini, A. Bazzani, M. Giovannozzi, E. Todesco, and W. Scandale, CERN Report No. SL/96-48 (AP), 1996.

[13] P. M. Ivanov, I. A. Koop, E. A. Perevedentsev, Yu. M. Shatunov, and I. B. Vasserman, in Proceedings of the 3rd Advanced ICFA Beam Dynamics Workshop, Novosibirsk, 1989 (BINP, Novosibirsk, Russia, 1989), p. 26.

[14] E. A. Perevedentsev and A. A. Valishev, in Proceedings of the 7th European Particle Accelerators Conference, EPAC2000, Vienna, http://accelconf.web.cern.ch/ AccelConf/e00/index.html, p. 1223. 